\title{
Compression of TW class laser pulses in a planar hollow waveguide for applications in strong-field physics ${ }^{\star}$
}

\author{
Amélie Jarnac ${ }^{2}$, Fernando Brizuela ${ }^{1}$, Christoph M. Heyl ${ }^{1}$, Piotr Rudawski ${ }^{1}$, Filippo Campi ${ }^{1}$, Byunghoon Kim ${ }^{1}$, \\ Linnea Rading ${ }^{1}$, Per Johnsson ${ }^{1}$, André Mysyrowicz ${ }^{2}$, Anne L'Huillier ${ }^{1}$, Aurélien Houard ${ }^{2}$, and Cord L. Arnold ${ }^{1}$, a \\ 1 Department of Physics, Lund University, P.O. Box 118, 22100 Lund, Sweden \\ 2 Laboratoire d'Optique Appliquée, ENSTA Paristech, École Polytechnique, CNRS, 91762 Palaiseau, France
}

Received 28 July 2014 / Received in final form 21 August 2014

Published online 16 December 2014

(C) The Author(s) 2014. This article is published with open access at Springerlink.com

\begin{abstract}
We demonstrate pulse post-compression of a TW class chirped pulse amplification laser employing a gas-filled planar hollow waveguide. A waveguide throughput of $80 \%$ is achieved for $50 \mathrm{~mJ}$ input pulse energy. Good focusability is found and after compression with chirped mirrors a pulse duration of sub-15 fs is measured in the beam center. Whereas a total energy efficiency of $\approx 70 \%$ should be achievable, our post-compressor currently delivers $20 \mathrm{~mJ}$ output pulse energy ( $\approx 40 \%$ efficiency), mostly limited by apertures of chirped mirrors and vacuum windows. The viability of the planar hollow waveguide compression scheme for applications in strong-field physics is demonstrated by generating high-order harmonics in a pulsed Ar gas cell.
\end{abstract}

\section{Introduction}

Few-cycle laser pulses, i.e. pulses so short that the pulse envelope encloses only a few cycles of the field, have become indispensable tools in optics and related sciences, such as High-order Harmonic Generation (HHG) [1], attosecond science [2], strong-field physics [3], and acceleration of particles [4]. While low-energy, few-cycle pulses can today routinely be obtained from Titanium:Sapphire (Ti:Saph) based ultrafast oscillators [5], the output of chirped pulse amplification (CPA) femtosecond lasers hardly reaches below 20 fs pulse duration, mostly due to gain-bandwidth-narrowing. Typical Ti:Saph based CPA lasers, found in research laboratories today, have pulse duration in the range of $25-100$ fs and pulse energies in the $\mathrm{mJ}$ to hundreds of $\mathrm{mJ}$ range. Common pulse post-compression techniques can hardly handle more than $10 \mathrm{~mJ}$ input pulse energy. Thus, there is a strong need for post-compression of energetic laser pulses.

The two most prominent schemes for pulse compression to the few-cycle regime are based on strong spectral broadening due to nonlinear propagation in either a noble gas filled hollow capillary [6] or in a self-guided filament [7] and subsequent compression with chirped mirrors. Due to ionization inside the capillary and nonlinear coupling to higher order capillary modes with stronger attenuation the output pulse energy of a hollow capillary compressor

\footnotetext{
* Contribution to the Topical Issue "X-ray Generation from Ultrafast Lasers", edited by Germán J. de Valcárcel, Luis Roso and Amelle Zaïr.

a e-mail: cord.arnold@fysik.lth.se
}

rarely exceeds a few mJ. Attempts to increase the energy output require advanced optical [8] or capillary engineering $[9,10]$, and may exhibit low efficiency [11].

The other scheme uses self-guiding in a laser filament, which forms as consequence of a dynamic equilibrium between self-focusing and plasma defocusing [12]. In certain conditions saturation of the Kerr-nonlinearity was suggested as an alternative effect to stabilize the filament [13]. Post-compression via filamentation in practice is limited to output pulse energies of about $1 \mathrm{~mJ}$, mainly due to the onset of multiple filamentation, spatial chirp, and angular dispersion [14,15]. Hence, only the center of the output beam is spectrally homogeneous and can be recompressed to a few-cycle pulse.

As alternative to hollow capillaries and filamentation, self-compression in the relativistic self-focusing regime has been suggested and demonstrated [16,17]. Whereas this approach might work even up to the PW power level, the energy efficiency usually is around 20\% [18].

A scheme that potentially scales to high pulse energy much more favorably than hollow capillaries and filamentation was proposed by Nurhuda et al. [19]. By use of gas-filled planar hollow waveguides for post-compression, the pulse energy can easily be up-scaled by increasing the beam size in one spatial direction, while keeping the intensity inside the waveguide at levels benefiting efficient self-phase modulation, but limiting ionization. In the first experimental realization it was recognized that modulational instability due to one-dimensional, small-scale selffocusing along the unguided waveguide direction could result in strong deterioration of the transverse beam profile 


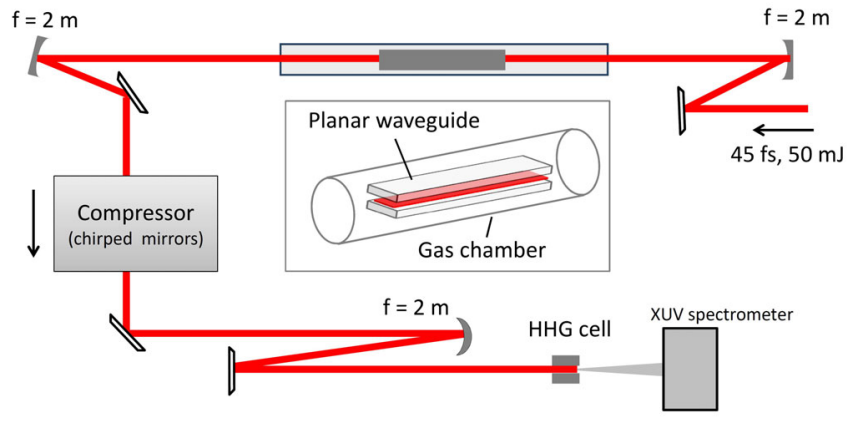

Fig. 1. Sketch of the experimental setup.

and in the extreme to break-up of the beam into several filaments [20]. Shortly after, some of the authors of this publication showed that by carefully controlling experimental parameters, such as gas pressure and waveguide length, a balance between spectral broadening and beam deterioration can be achieved and demonstrated compressed pulses at the $10 \mathrm{~mJ}$ level with good focusability $[21,22]$. Furthermore, a theoretical model describing a stability regime for energy up-scaling of the planar hollow waveguide compression scheme was introduced [23]. The results from these early experiments were summarized in an article [24], which discusses in detail how the detrimental transverse dynamics inside the waveguide can be balanced in order to support energies exceeding the current limitations in pulse post-compression with hollow capillaries and filamentation.

In the current work, we present pulse post-compression of a TW class CPA laser in a gas-filled planar hollow waveguide with up to $50 \mathrm{~mJ}$ input pulse energy. While the waveguide throughput can be as high as $80 \%$, the total efficiency of the compression setup currently is $\approx 40 \%$ $(20 \mathrm{~mJ})$, mainly limited by the apertures of chirped mirrors and vacuum windows. The compressed pulses are characterized in terms of beam profile, focusability, and pulse duration. We demonstrate the suitability of such pulses for applications in high-field physics by performing HHG in a pulsed Ar gas cell. The harmonic cut-off is significantly increased compared to that obtained with longer driving pulses.

\section{Experiments and discussion}

\subsection{Setup}

The experimental setup is sketched in Figure 1. Pulses of up to $50 \mathrm{~mJ}$ pulse energy at $10 \mathrm{~Hz}$ repetition rate and duration of $45 \mathrm{fs}$, centered around $800 \mathrm{~nm}$ are focused with a cylindrical focusing mirror $(f=2 \mathrm{~m})$ onto the input of the planar hollow waveguide. The pulse energy can be adjusted by a $\lambda / 2$-plate and a polarizer installed before the grating compressor of the CPA chain. The beam size before focusing is $\approx 25 \mathrm{~mm}$ (FWHM). The waveguide consists of two parallel, highly polished glass slabs (Plan Optik) made of Borofloat with dimensions $500 \times 40 \times 10 \mathrm{~mm}^{3}$ with a gap of $2 a=127 \mu \mathrm{m}$ between them. A homogeneous gap size is achieved by putting narrow spacers, made of a polycarbonate foil (Precision Brand), along the long sides of the waveguide. This reduces the usable transverse aperture to $\approx 36 \mathrm{~mm}$. The size of the gap is matched to the width of the lineshaped laser focus at the waveguide entrance $w_{y} \approx 0.735 a$, where $w_{y}$ is the Gaussian beam size $\left(1 / e^{2}\right)$; this condition facilitates maximum coupling efficiency into the fundamental waveguide mode. We make the arbitrary choice in the following to call the unguided (free) waveguide dimension the $x$-direction and the guided the $y$-direction. The waveguide is located inside a gas cell with thin $(2.2 \mathrm{~mm})$, broadband anti-reflection coated fused silica windows at both ends. The gas cell is approximately $3 \mathrm{~m}$ long in order to minimize detrimental nonlinear interaction with the windows. After the gas cell, the output beam of the waveguide is collimated with an identical cylindrical mirror and passed through a chirped mirror compressor. The beam is then sent to a HHG setup and harmonic spectra are recorded with a flat-field, XUV photon spectrometer [25].

The gas cell, in which the waveguide is located, can be filled with $\mathrm{Ar}$ at pressures up to one atmosphere. The waveguide throughput is higher than $80 \%$ in vacuum $(86 \%$ in theory), proving good coupling to the fundamental mode. It does not decrease up to 400 mbar of Ar, indicating that losses due to ionization and nonlinear coupling to higher order modes are small. Beyond 500 mbar the transverse mode breaks up into a number of filaments, unusable for pulse compression. At the same time, the throughput drops, indicating increased losses due to stronger nonlinear propagation effects and stronger ionization.

\subsection{Spectral broadening, beam profile, and focusability}

Figure 2a shows the spectra observed at the input and output of the waveguide for pulse energy of $50 \mathrm{~mJ}$ in vacuum, at 200, and 400 mbar of Ar. For the evacuated gas cell, the spectrum slightly broadens, probably due to residual parasitic nonlinear interaction with the windows. When the gas cell is filled with 400 mbar of Ar, an about four-fold increase of the spectral width is observed with the spectral wings spanning from 700 to $900 \mathrm{~nm}$. The broadening is only slightly blue-shifted, identifying self-phase modulation as the main source of spectral broadening with only small contribution from plasma related blue-shifting [26]. The spectrum obtained at 400 mbar supports a transformlimited pulse duration of $13 \mathrm{fs}$. The spectra were measured at the center of the output beam. It should be noted that along the unguided waveguide direction, spectral broadening is strongest at the beam center and weaker at the wings. Inhomogeneous spectral broadening leads to different transverse pulse duration after compression, with the shortest pulse obtained at the beam center and longer pulse duration at the wings. This effect was investigated in detail in reference [24].

Transverse beam profile and focusability are important criteria for pulse compression in planar hollow waveguides. If the waveguide output is not focusable to a small spot size, pulse post-compression will not result in an increase of the peak intensity. As was discussed in detail in reference [24], the focusability in the non-guided 

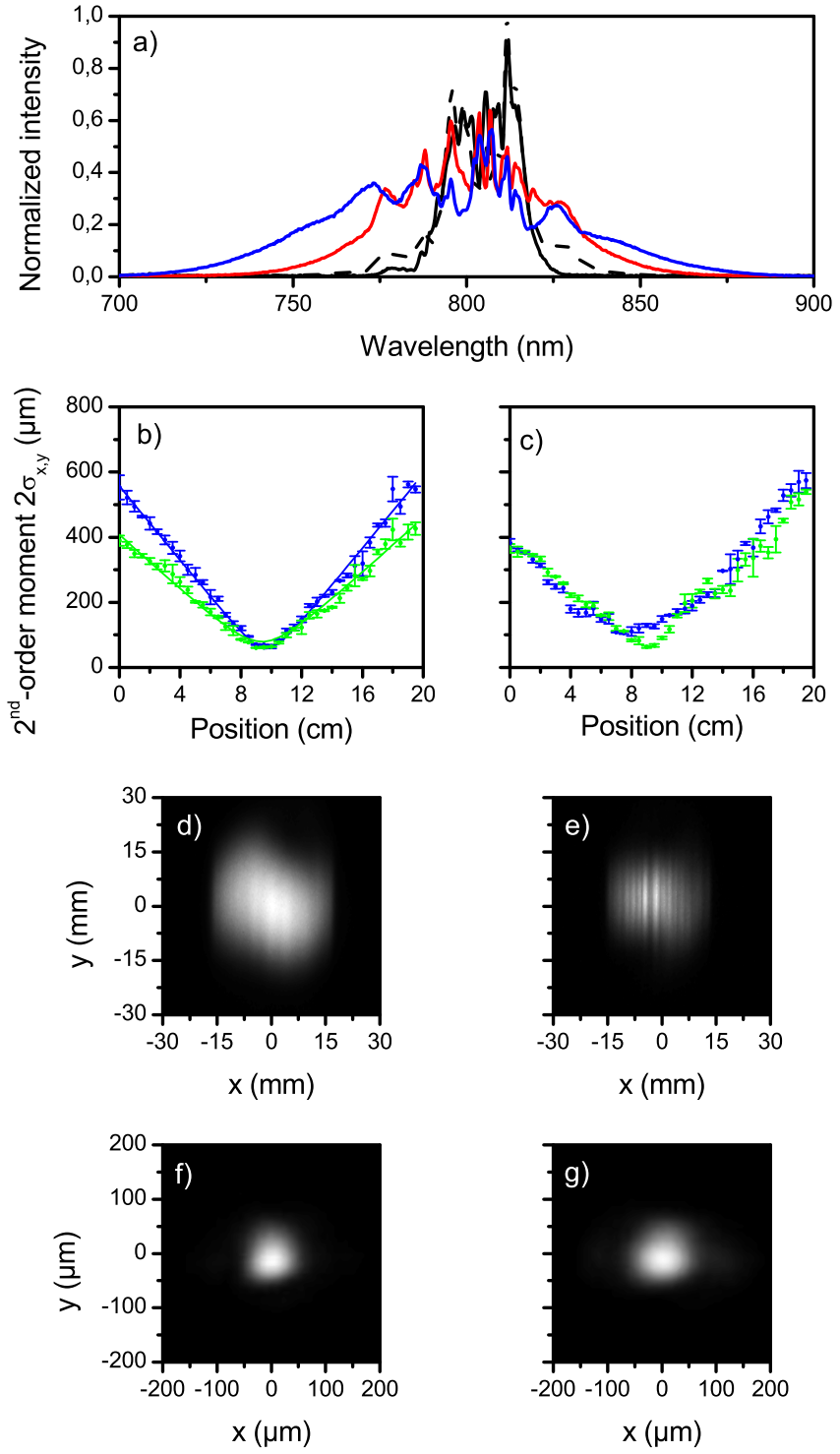

Fig. 2. (a) Spectra observed at the waveguide input (solid black) and output (dashed black) for vacuum and for 200 (red) and 400 mbar (blue) of Ar. (b) and (c) Focusability of the guided (green) and free (blue) waveguide directions, illustrated in terms of beam caustics for the waveguide under vacuum (b) and 400 mbar of $\operatorname{Ar}$ (c). $M^{2}$-fits are plotted in (b). The far-field output mode from the waveguide and the near-field focal spot after focusing with a $f=2 \mathrm{~m}$ lens are shown for the case of vacuum in panels (d) and (f) and for 400 mbar of Ar in panels (e) and (g).

waveguide direction inevitably diminishes; first, due to the transversely inhomogeneous spectrum and second, due to a pulse front deformation resulting from the beam center propagating slower (due to the Kerr-effect) than the wings. The second effect essentially introduces an astigmatism (to first-order approximation), which however can easily be compensated by translating the collimating cylindrical mirror [24]. Finally, modulational instability from one-dimensional self-focusing deteriorates the mode along the unguided waveguide direction.
The far-field output modes, recorded with a camera at the position of the collimating cylindrical mirror, are shown in Figures 2d and 2e for vacuum and 400 mbar of Ar, respectively. A modulation along the unguided waveguide direction is evident for the latter case (Fig. 2e). With increasing pressure of Ar the transverse mode further deteriorates and finally breaks up into a number of filaments at $\approx 500$ mbar, unusable for pulse compression. As selfphase modulation and self-focusing are the spectral and spatial manifestations of the Kerr-nonlinearity, transverse beam break-up ultimately limits the maximum spectral broadening and thus the shortest achievable compressed pulse duration [24]. Modulational instability in principle results from noise, but in practice starts from small imperfections in the transverse beam profile. To delay the onset of break-up, the laser beam profile was spatially filtered before the grating compressor of our CPA chain. The sharp cuts at the sides of far-field output modes along the unguided waveguide direction (Figs. 2d and 2e) originate from clipping of the input beam at the waveguide spacers.

The focusability is investigated by focusing the beam, after careful attenuation with reflections from uncoated wedges, with a thin lens $(f=2 \mathrm{~m})$ onto the chip of a CCD camera. The camera is moved along the optical axis to record the focal spot in the vicinity of the geometrical focus. The beam size is extracted from the images by computing second order moments $\sigma_{x, y}$. For Gaussian beams $2 \sigma_{x, y}=w_{x, y}$, where $w_{x, y}$ is the Gaussian beam radius $\left(1 / e^{2}\right)$. Figures $2 \mathrm{~b}$ and $2 \mathrm{c}$ show the beam size $2 \sigma_{x, y}$ as a function of position for the guided and the free waveguide directions for the evacuated waveguide (b) and filled with 400 mbar of Ar (c). Indeed, the beam sizes obtained by moment theory are very close to those resulting from fitting a Gaussian profile (not shown), indicating that the focal spot is single-peaked and close to Gaussian shape. The focusability in terms of a $M^{2}$-parameter is obtained by fitting a parabola to the beam caustic (beam size vs. propagation distance) [27]. For the case of vacuum, $M_{y}^{2} \approx 1.28$ and $M_{x}^{2} \approx 1.32$ in the guided and free waveguide directions are obtained, respectively. For a filling pressure of 400 mbar of Ar, the focusability in the guided direction remains approximately unchanged, while in the free direction the minimum beam size increases by a factor of $\approx 1.6$. At the same time, the focal position moves towards the lens, exhibiting a slight astigmatism, which however is easily removed by moving the collimation mirror [24]. The near-field focal spots for vacuum and 400 mbar are shown in Figures $2 \mathrm{f}$ and $2 \mathrm{~g}$, respectively. It can clearly be observed that the beam size remains approximately the same in the guided direction, while it increases slightly in the free waveguide direction. It is interesting to note that the transverse mode structure in the far-field (Fig. 2e) does not reduce the focusable intensity significantly, as was shown in reference [24].

\subsection{Pulse duration measurement}

The waveguide output is compressed using chirped mirrors from Layertec and Venteon, respectively. 

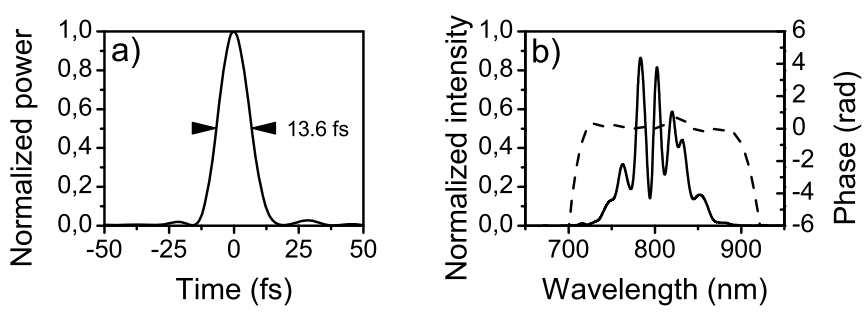

Fig. 3. Temporal pulse profile (a) and spectral intensity and phase (b) obtained from SPIDER measurement.

While $\approx 40 \mathrm{~mJ}$ pulses are obtained at the output of the waveguide (50 $\mathrm{mJ}$ at the input), the compressed pulse energy available for experiments is $\approx 20 \mathrm{~mJ}$. The losses are mostly due to clipping at the limited apertures of vacuum windows and chirped mirrors. The compressed pulses are characterized with a commercial, few-cycle SPIDER (Venteon). Dispersion can be fine-tuned by transmitting the beam through a pair of anti-reflection coated fused silica wedges. Note that the wedges can only be put after strongly attenuating the beam, otherwise filaments form inside. Thus, for actual experiments with the waveguide output, dispersion has to be coarsely adjusted by the number of bounces from the chirped mirrors without fine dispersion control.

Figure 3 shows a SPIDER measurement in optimized conditions for $50 \mathrm{~mJ}$ pulse energy at the waveguide input and 400 mbar of Ar. Individual measurements exhibit a large spread in retrieved pulse duration due to shot-toshot pulse energy fluctuations, typical for low repetition rate TW class lasers. We performed ten SPIDER measurements, with retrieved duration spreading from 13.4 fs to $17 \mathrm{fs}$; the average pulse duration was $14.3 \mathrm{fs}$. The retrieved spectral phase (Fig. $3 \mathrm{~b}$ ) is reasonably flat, indicating that the chirped mirror compressor is well suited to compress the waveguide output. The pulse duration was measured in the center of the beam. Due to less spectral broadening, the compressed pulse duration becomes longer towards the edges of the waveguide. It was shown before, both experimentally and numerically, that for a Gaussian transverse beam profile, about $70 \%$ of the compressed pulse energy falls within a pulse duration interval of 1.5 times the center pulse duration [24]. By translating the conclusions from [24] to our current data, we can estimate that the $20 \mathrm{~mJ}$ pulses available for experiments have an average pulse duration of $\approx 22 \mathrm{fs}$. By clipping the beam along the free direction, one can select shorter pulses with lower energy, e.g. $\approx 14 \mathrm{~mJ}$ with an average pulse duration of $\approx 17 \mathrm{fs}$.

\subsection{High-order harmonic generation}

After having demonstrated strong spectral broadening, high throughput, good focusability, and sub-15 fs center pulse duration, we used the compressed pulses to drive high-order harmonic generation. In particular, we compared the generated XUV spectrum with the one obtained with longer pulses, circumventing the planar hol-

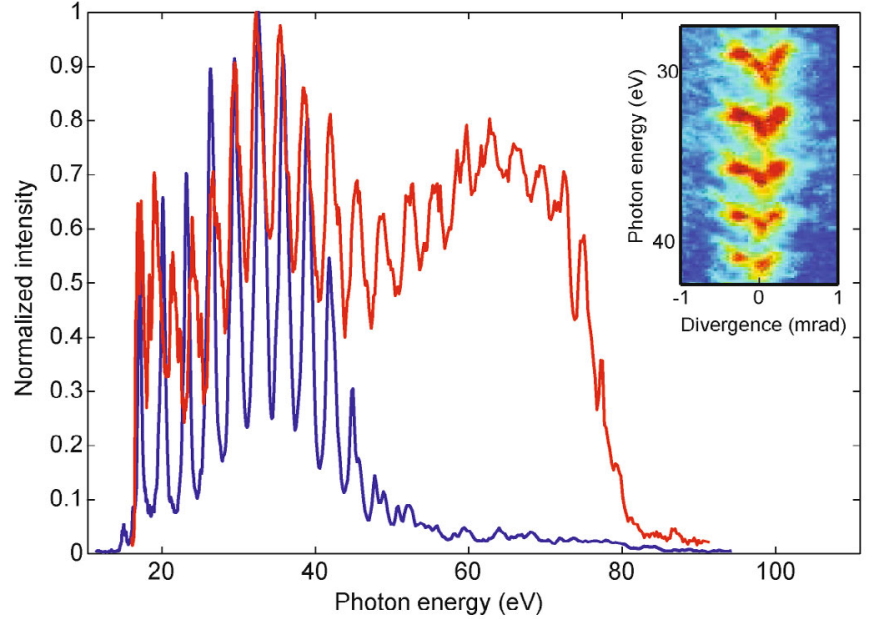

Fig. 4. HHG spectrum in Ar with (red) and without (blue) waveguide compressor at $\approx 20 \mathrm{~mJ}$ pulse energy. The inset shows the spatial shape for a range of harmonics generated from the waveguide compressed pulses.

low waveguide compressor, in similar conditions. HHG requires high field intensity (exceeding $10^{14} \mathrm{~W} \mathrm{~cm}^{-2}$ ) and thus serves as a sensitive measure to demonstrate that post-compression with a planar hollow waveguide can provide short pulses, good focusability, and high field intensity. HHG essentially is a strong-field effect and is most easily explained by the semi-classical three-step model [28]. The HHG spectrum is comprised of discrete odd-order harmonics of the driving field frequency, whereas the spectral width of individual harmonics decreases with increasing number of half-cycles contributing to harmonic generation. In the single-atom response picture, the highest energy $E_{\max }$ in a HHG spectrum is related to the driving field intensity by the cut-off law $E_{\max }=3.17 U_{P}+I_{P}[29]$, where $U_{P}$ is the ponderomotive energy (average energy of an electron in an AC field with $\left.U_{P} \propto I_{\max }\right), I_{P}$ is the ionization potential of the gas, and $I_{\text {max }}$ is the peak intensity of the driving field. In our experimental conditions, the cut-off energy is rather determined by macroscopic phase-matching and propagation effects.

Strong ionization of the gas medium leads both to reshaping of the fundamental field due to plasma-defocusing as well as to termination of HHG due to plasma-related unfavorable phase-matching conditions. Thus generating with shorter pulses, two main effects should be observed: first, a broadening of the individual harmonics due to fewer half-cycles contributing to the generation process and second, a higher cut-off energy due to higher peak intensity as well as due to plasma-related effects setting in later for shorter pulses.

The compressed waveguide output is loosely focused with a spherical mirror $(f=2 \mathrm{~m})$ into an Ar gas cell. Figure 4 shows HHG spectra obtained in two different situations, using long pulses $\approx 45 \mathrm{fs}$, circumventing the waveguide setup (blue), or using the waveguide compressor with 400 mbar of Ar (red). The pulse energy in both cases is $\approx 20 \mathrm{~mJ}$ and the beam sizes before 
focusing are comparable. With the waveguide compressor the individual harmonics become broader and the cut-off energy increases, indicating that the pulses are significantly shorter and more intense. Furthermore, the harmonics feature rich transverse spatial structure (see inset), where each of the harmonics exhibits a spectrally asymmetric ' $V$ '-shape with higher central frequency onaxis as compared to the wings. Such behavior is not observed with the long pulses. It might result from blueshifting of the fundamental on-axis due to high ionization yield [30]. In addition, the transversely varying spectrum and pulse duration of the waveguide output can potentially lead to complicated spatio-temporal couplings in combination with nonlinear propagation and the strongly nonlinear dynamics of the HHG process itself [31]. Although interesting in itself, a detailed interpretation of the particular shape of the harmonics is beyond the scope of this article.

\section{Conclusion and outlook}

In conclusion, we have demonstrated that planar hollow waveguides can be used to post-compress typical TW CPA lasers, which are currently operated in laboratories around the world. In this work $50 \mathrm{~mJ}$ input pulses could be compressed to a center pulse duration of sub-15 fs with a total pulse energy of $20 \mathrm{~mJ}$. The viability of the scheme for applications in strong-field physics was proven by driving high-order harmonic generation with the compressed waveguide output. The total throughput is currently limited by the sizes of chirped mirrors and vacuum windows. A carefully designed chirped mirror compressor should in the future allow for $70 \%$ total energy efficiency (waveguide-input-to-compressed-output). Furthermore, considering the transverse variation in compressed pulse duration inherent to the planar hollow waveguide scheme [24], pulse duration shorter than 1.5 times the center pulse duration can be obtained with a total energy efficiency of $\approx 50 \%$. Although we could not achieve pulse durations significantly below 15 fs, the pulse energy range presented here currently is and most likely will remain not easily accessible with competing compression schemes, such as hollow capillaries or filamentation. Furthermore, the planar hollow waveguide still bears great potential for energy up-scaling.

Up-scaling can most easily be discussed in terms of the $B$-integral acquired by the beam during nonlinear propagation inside the waveguide,

$$
B=\int_{z=0}^{L} k_{0} I(z) n_{2} P z d z \approx k_{0} I_{0} n_{2} P L
$$

where $k_{0}$ is the wavenumber at the carrier frequency, $I_{0}$ is the intensity at the waveguide input, $n_{2}$ is the nonlinear refractive index for Ar at atmospheric pressure, $P$ is the gas pressure in atmospheres, and $L$ is the length of the waveguide. For the current experiment, the $B$-integral accumulated in the waveguide is $B \approx 8$. While a higher $B$-integral would lead to stronger spectral broadening, inevitably the transverse beam profile would break into filaments, resulting in an unusable beam. Consequently, energy up-scaling must be achieved, while keeping the $B$-integral constant. Increasing the beam size in the unguided direction and using larger waveguides provides a trivial way to up-scale the pulse energy. Further control knobs are intensity, pressure, length of the waveguide, and type of gas. The intensity however should be kept below a level of strong ionization $\left(\approx 7 \times 10^{13} \mathrm{~W} \mathrm{~cm}^{-2}\right.$ in the current experiment) to avoid plasma-related absorption and nonlinear coupling to higher waveguide modes. For $\mathrm{Ne}$ and He as filling gases the pulse energy can be increased by factors of $\approx 3$ and $\approx 5$, respectively, while keeping similar relative ionization $[32]$. The lower nonlinearity $(\approx 7$ times for $\mathrm{Ne}$ and $\approx 33$ times for $\mathrm{He}$ ) has to be compensated by higher pressure inside the waveguide. Considering the degrees of freedom sketched above, an up-scaling to input pulse energies in the range of hundreds of mJ seems feasible, offering a possibility to expand the concept of pulse post-compression to lasers with tens of TW peak power.

This research was supported by the European Research Council (ALMA, PALP), the Swedish Research Council, the Crafoord Foundation, the Knut and Alice Wallenberg Foundation, and the Swedish Foundation for Strategic Research. A.J. and A.H. acknowledge support from the French DGA, the European Science Foundation (SILMI), and the European Cooperation in Science and Technology (COST).

\section{References}

1. A. L'Huillier, T. Auguste, Ph. Balcou, B. Carre, P. Monot, P. Salieres, C. Altucci, M.B. Gaarde, J. Larsson, E. Mevel, T. Starczewski, S. Svanberg, C.G. Wahlstrom, R. Zerne, K.S. Budil, T. Ditmire, M.D. Perry, J. Nonlinear Opt. Phys. Mater. 4, 647 (1995)

2. F. Krausz, M. Ivanov, Rev. Mod. Phys. 81, 163 (2009)

3. T. Brabec, F. Krausz, Rev. Mod. Phys. 72, 545 (2000)

4. E. Esarey, C.B. Schroeder, W.P. Leemans, Rev. Mod. Phys. 81, 1229 (2009)

5. S. Rausch, T. Binhammer, A. Harth, E. Schulz, M. Siegel, U. Morgner, Opt. Express 17, 20282 (2009)

6. M. Nisoli, S. De Silvestri, O. Svelto, R. Szipöcs, K. Ferencz, C. Spielmann, S. Sartania, F. Krausz, Opt. Lett. 22, 522 (1997)

7. C. Hauri, W. Kornelis, F. Helbing, A. Heinrich, A. Couairon, A. Mysyrowicz, J. Biegert, U. Keller, Appl. Phys. B 79, 673 (2004)

8. X. Chen, A. Malvache, A. Ricci, A. Jullien, R. LopezMartens, Fib. Opt. 21, 198 (2011)

9. S. Bohman, A. Suda, M. Kaku, M. Nurhuda, T. Kanai, S. Yamaguchi, K. Midorikawa, Opt. Express 16, 10684 (2008)

10. F. Böhle, M. Kretschmar, A. Jullien, M. Kovacs, M. Miranda, R. Romero, H. Crespo, U. Morgner, P. Simon, R. Lopez-Martens, T. Nagy, Las. Phys. Lett. 11, 095401 (2014)

11. C. Fourcade Dutin, A. Dubrouil, S. Petit, E. Mével, E. Constant, D. Descamps, Opt. Lett. 35, 253 (2010)

12. A. Couairon, A. Mysyrowicz, Phys. Rep. 441, 47 (2007) 
13. P. Béjot, E. Hertz, J. Kasparian, B. Lavorel, J.-P. Wolf, O. Faucher, Phys. Rev. Lett. 106, 243902 (2011)

14. A. Couairon, J. Biegert, C.P. Hauri, W. Kornelis, F.W. Helbing, U. Keller, A. Mysyrowicz, J. Mod. Opt. 53, 75 (2006)

15. A. Zair, A. Guandalini, F. Schapper, M. Holler, J. Biegert, L. Gallmann, A. Couairon, M. Franco, A. Mysyrowicz, U. Keller, Opt. Express 15, 5394 (2007)

16. C. Ren, B.J. Duda, R.G. Hemker, W.B. Mori, T. Katsouleas, T. Antonsen, P. Mora, Phys. Rev. E 63, 026411 (2001)

17. J. Faure, Y. Glinec, J.J. Santos, F. Ewald, J.-P. Rousseau, S. Kiselev, A. Pukhov, T. Hosokai, V. Malka, Phys. Rev. Lett. 95, 205003 (2005)

18. A. Pipahl, E.A. Anashkina, M. Toncian, T. Toncian, S.A. Skobelev, A.V. Bashinov, A.A. Gonoskov, O. Willi, A.V. Kim, J. Phys.: Conf. Ser. 414, 012011 (2013)

19. M. Nurhuda, A. Suda, S. Bohman, S. Yamaguchi, K. Midorikawa, Phys. Rev. Lett. 97, 153902 (2006)

20. J. Chen, A. Suda, E.J. Takahashi, M. Nurhuda, K. Midorikawa, Opt. Lett. 33, 2992 (2008)

21. S. Akturk, C.L. Arnold, B. Zhou, A. Mysyrowicz, Opt. Lett. 34, 1462 (2009)

22. S. Chen, A. Jarnac, A. Houard, Y. Liu, C.L. Arnold, B. Zhou, B. Forestier, B. Prade, A. Mysyrowicz, J. Opt. Soc. Am. B 28, 1009 (2011)
23. C.L. Arnold, S. Akturk, M. Franco, A. Couairon, A. Mysyrowicz, Opt. Express 17, 11122 (2009)

24. C.L. Arnold, B. Zhou, S. Akturk, S. Chen, A. Couairon, A. Mysyrowicz, N. J. Phys. 12, 073015 (2010)

25. P. Rudawski, C.M. Heyl, F. Brizuela, J. Schwenke, A. Persson, E. Mansten, R. Rakowski, L. Rading, F. Campi, B. Kim, P. Johnsson, A. L'Huillier, Rev. Sci. Instrum. 84, 073103 (2013)

26. A. Brodeur, S.L. Chin, Phys. Rev. Lett. 80, 4406 (1998)

27. A. Siegman, OSA TOPS 17, 184 (1998)

28. P.B. Corkum, Phys. Rev. Lett. 71, 1994 (1993)

29. J.L. Krause, K.J. Schafer, K.C. Kulander, Phys. Rev. Lett. 68, $3535(1992)$

30. M.B. Gaarde, J.L. Tate, K.J. Schafer, J. Phys. B 41, 132001 (2008)

31. A. Dubrouil, O. Hort, F. Catoire, D. Descamps, S. Petit, E. Mével, V.V. Strelkov, E. Constant, Nat. Commun. 5, 4637 (2014)

32. A.M. Perelomov, V.S. Popov, M.V. Terent'ev, JETP 23, 924 (1966)

Open Access This is an open access article distributed under the terms of the Creative Commons Attribution License (http://creativecommons.org/licenses/by/4.0), which permits unrestricted use, distribution, and reproduction in any medium, provided the original work is properly cited. 